TRANSACTIONS OF THE

AMERICAN MATHEMATICAL SOCIETY

Volume 359, Number 6, June 2007, Pages 2539-2558

S 0002-9947(07)04274-2

Article electronically published on January 25, 2007

\title{
THE REAL COHOMOLOGY OF VIRTUALLY NILPOTENT GROUPS
}

\author{
KAREL DEKIMPE AND HANNES POUSEELE
}

\begin{abstract}
In this paper we present a method to compute the real cohomology of any finitely generated virtually nilpotent group. The main ingredient in our setup consists of a polynomial crystallographic action of this group. As any finitely generated virtually nilpotent group admits such an action (which can be constructed quite easily), the approach we present applies to all these groups. Our main result is an algorithmic way of computing these cohomology spaces. As a first application, we prove a kind of Poincaré duality (also in the nontorsion free case) and we derive explicit formulas in the virtually abelian case.
\end{abstract}

\section{INTRODUCTION}

In [13, Section 8] it was shown that the cohomology of a compact and complete affinely flat manifold with a nilpotent fundamental group can be computed as the cohomology of a certain complex of polynomial differential forms. Any such manifold $M$ can be constructed as a quotient space $\rho(G) \backslash \mathbb{R}^{n}$, where $\rho: G \cong \pi_{1}(M) \rightarrow$ $\operatorname{Aff}\left(\mathbb{R}^{n}\right)$ defines a cocompact, free and properly discontinuous action of $G$ on $\mathbb{R}^{n}$ (see [1]). Here $\operatorname{Aff}\left(\mathbb{R}^{n}\right)$ denotes the group of invertible affine maps of $\mathbb{R}^{n}$. We generalize this affine set-up by considering group actions $\rho: G \rightarrow \mathrm{P}\left(\mathbb{R}^{n}\right)$, where $\mathrm{P}\left(\mathbb{R}^{n}\right)$ is the group of all polynomial diffeomorphisms of $\mathbb{R}^{n}$. A map $p: \mathbb{R}^{n} \rightarrow \mathbb{R}^{n}$ belongs to $\mathrm{P}\left(\mathbb{R}^{n}\right)$ iff $p$ is bijective and both $p$ and $p^{-1}$ are expressed by polynomials. As group actions will play a crucial role in this paper, we introduce the following notions.

Definition 1.1. Let $\mathcal{H}\left(\mathbb{R}^{n}\right)$ be the group of homeomorphisms of $\mathbb{R}^{n}$. An action $\rho: G \rightarrow \mathcal{H}\left(\mathbb{R}^{n}\right)$ is said to be crystallographic iff $G$ acts properly discontinuously and cocompactly on $\mathbb{R}^{n}$. If $\rho(G) \subseteq \operatorname{Aff}\left(\mathbb{R}^{n}\right)$, the action is called an affine crystallographic action, and if $\rho(G) \subseteq \mathrm{P}\left(\mathbb{R}^{n}\right)$ we use the term polynomial crystallographic action. The image $\rho(G)$ is referred to as a (possibly affine or polynomial) crystallographic group.

Of course this terminology is based on the same terminology for actions $\rho: G \rightarrow$ $\operatorname{Isom}\left(\mathbb{R}^{n}\right)$, with $\operatorname{Isom}\left(\mathbb{R}^{n}\right)$ the group of isometries of $n$-dimensional Euclidean space.

We can translate the result of [13 to the group level, by stating that the cohomology of a torsion-free nilpotent affine crystallographic group can be obtained as the cohomology of a certain complex of polynomial differential forms. In fact, given an affine crystallographic action $\rho: N \rightarrow \operatorname{Aff}\left(\mathbb{R}^{n}\right)$ of a finitely generated

Received by the editors February 3, 2005.

2000 Mathematics Subject Classification. Primary 20J06, 57T15.

The second author is a Research Assistant of the Fund for Scientific Research-Flanders (F.W.O.).

(C) 2007 American Mathematical Society Reverts to public domain 28 years from publication 
torsion-free group $N$ (e.g. by the images of a set of generators of $N$ ), the result of Fried, Goldman and Hirsch in [13] implies there is an algorithm to compute the cohomology groups $H_{\text {triv }}^{*}(N, \mathbb{R})$.

Unfortunately, there are some problems with this approach. The major problem is the fact that not all finitely generated torsion-free nilpotent groups admit such an affine crystallographic action (see [3, 8, and 7] for examples). Moreover, even if such a group does admit an affine crystallographic action, there is no practical method, and certainly no algorithm, to find it.

In this paper we extend the approach of Fried, Goldman and Hirsch in two directions. First of all, we will not restrict ourselves to affine crystallographic actions, but to polynomial crystallographic actions of bounded degree. (A polynomial crystallographic action $\rho: N \rightarrow \mathrm{P}\left(\mathbb{R}^{n}\right)$ is said to be of bounded degree, if there exists an integer $M$ such that for all $g \in G$, the degree of the polynomial map $\rho(g)$ is bounded above by $M$.) This generalization completely overcomes the problems mentioned above, since any finitely generated nilpotent-by-finite group (in fact, any polycyclic-by-finite group) admits a polynomial crystallographic action, which can be determined easily (e.g. there is an algorithmic way to construct a polynomial crystallographic action from a presentation of the group, see [12, 10, [11]). Secondly, our methods do not only apply to finitely generated torsion-free nilpotent groups, but are also valid for all finitely generated nilpotent-by-finite groups, even if there is torsion.

The methods we develop in this article allow us to compute the Betti numbers of all virtually nilpotent groups, whereas results somehow familiar to ours (as can be found in for instance [20, pp. 189-209]) seem to be restricted to the torsion-free case. As an easy example we present explicit formulas for the computation of these Betti numbers for all crystallographic groups.

\section{Cohomology of $K(G, 1)$-Spaces}

Let $X$ be a contractible, connected and locally arcwise connected topological space. When a group $G$ acts freely and properly discontinuously via homeomorphisms on $X$ (denoted by a group homomorphism $\rho: G \rightarrow \mathcal{H}(X)$ ), the quotient space $Y=G \backslash X$ is a $K(G, 1)$-space. The well-known Eilenberg-Mac Lane theorem then relates the singular cohomology of $Y$ with coefficients in an abelian group $A$ (see for instance [16]) to the group cohomology of $G$, with coefficients in $A$ considered as a trivial $G$-module (see for instance [2], 6]).

Theorem 2.1. $H_{\text {sing }}^{*}(Y, A) \cong H_{\text {triv }}^{*}(G, A)$. Moreover, this is a ring isomorphism for the cup product ring structures.

As we will need the exact nature of the isomorphism between those two cohomology groups, we describe it here in detail. Let $C_{*}(X)$ denote the cubical singular chain complex of $X$. Any continuous map $f: X \rightarrow X$ induces a chain endomorphism $f_{\#}$ of $C_{*}(X)$, so the action of $G$ on $X$ induces an action on the chain complex $C_{*}(X)$ given by

$$
\rho(-)_{\#}: G \rightarrow \operatorname{Aut}\left(C_{*}(X)\right): g \mapsto \rho(g)_{\#} .
$$

This action turns $C_{*}(X)$ into a free resolution of $\mathbb{Z}$ as a trivial $G$-module, from which we can compute $H_{\text {triv }}^{*}(G, A)$ by applying the functor $\operatorname{Hom}_{\mathbb{Z} G}(-, A)$. The isomorphism between $H_{\text {triv }}^{*}(G, A)$ and $H_{\text {sing }}^{*}(Y, A)=H^{*}\left(\operatorname{Hom}_{\mathbb{Z}}\left(C_{*}(Y), A\right)\right)$ is given 
by the mapping $p^{*}: H_{\text {sing }}^{*}(Y, A) \rightarrow H_{\text {triv }}^{*}(G, A)$ induced on cohomology by the projection $p: X \rightarrow Y$. Written explicitly,

$$
p^{*}(f): C_{*}(X) \rightarrow A: u \mapsto\left(f \circ p_{\#}\right)(u)
$$

for every $f \in \operatorname{Hom}_{\mathbb{Z}}\left(C_{*}(Y), A\right)$.

In case $X$ is a differentiable manifold and $G$ acts freely and properly discontinuously via diffeomorphisms (still denoted by $\rho: G \rightarrow \mathcal{D}(X)$ ), the quotient space $Y$ is also a manifold. The inclusion $i: C_{*}^{D}(Y) \rightarrow C_{*}(Y)$ of the chain complex $C_{*}^{D}(Y)$ of the differential singular (co)homology of $Y$ in the chain complex $C_{*}(Y)$ of the usual singular (co)homology of $Y$ induces an isomorphism on cohomology

$$
H^{*}(i): H_{\text {sing }}^{*}(Y, A) \rightarrow H_{\text {diff sing }}^{*}(Y, A) .
$$

The deRham theorem then relates this differential singular cohomology of $Y$ with coefficients in $\mathbb{R}$ to its deRham cohomology, which is the cohomology of the cochain complex $\Omega^{*}(Y)$ of differential forms on $Y$.

Theorem 2.2. $H_{\text {diff sing }}^{*}(Y, \mathbb{R}) \cong H_{d R}^{*}(Y)$ if $Y$ is paracompact. Moreover, this is a ring isomorphism: the ring structure arising from the cup product on singular cohomology corresponds to the the ring structure arising from the exterior product of forms.

The isomorphism of the cohomology spaces in the theorem above is induced by the cochain morphism

$$
\Phi: \Omega^{*}(Y) \rightarrow \operatorname{Hom}_{\mathbb{Z}}\left(C_{*}^{D}(Y), \mathbb{R}\right): \omega \mapsto \int_{\ldots} \omega,
$$

where the integral is a linear map from $C_{p}^{D}(Y)$ to $\mathbb{R}$ defined on a given differentiable singular $p$-cube $T:[0,1]^{p} \rightarrow Y$ by

$$
\int_{T} \omega=\int_{[0,1]^{p}} f d x_{1} d x_{2} \ldots d x_{p}
$$

when $T^{*}(\omega)=f d x_{1} \wedge d x_{2} \wedge \ldots \wedge d x_{p}$.

Putting all the results from this section together, we obtain the following.

Conclusion. Let $X$ be a contractible, connected and locally connected manifold and let $G$ be a group acting freely and properly discontinuously on $X$ via diffeomorphisms such that the quotient manifold $Y=G \backslash X$ is paracompact. Then

$$
H_{\text {triv }}^{*}(G, \mathbb{R}) \cong H_{d R}^{*}(Y) \text {. }
$$

Moreover, the ring structures of both cohomology spaces correspond under the isomorphism.

\section{Cohomology of $\mathcal{J}$-Groups}

In our context, a $\mathcal{J}$-group is a finitely generated torsion-free nilpotent group. According to the previous section, computing the cohomology of a $\mathcal{J}$-group $N$, with trivial coefficient module $\mathbb{R}$, is equivalent to the computation of the deRham cohomology of a $K(N, 1)$-manifold. To obtain such a $K(N, 1)$-manifold, we need to construct a suitable action of $N$ on a contractible space. We will put together the results obtained from two different constructions of such a $K(N, 1)$-manifold and work out an algorithmic way to compute this deRham cohomology. 


\subsection{Constructing $K(N, 1)$-manifolds.}

Lie group approach. Let $G$ be the Mal'cev completion of $N$. Then $G$ is diffeomorphic to some $\mathbb{R}^{n}$, so it is contractible, connected and locally arcwise connected. As $N$ is a lattice in $G$, the action of $N$ on $G$ given by left translations

$$
\mathrm{L}: N \rightarrow \mathcal{D}(G): n \mapsto \mathrm{L}_{n}, \quad \text { with } L_{n}(g)=n g \text { for all } g \in G,
$$

is free and properly discontinuous (see for instance [18]). The quotient manifold $N \backslash G$ is a compact $K(N, 1)$-manifold, so we know that

$$
H_{\text {triv }}^{*}(N, \mathbb{R}) \cong H_{d R}^{*}(N \backslash G) .
$$

The pullback $p^{*}$ of the projection $p: G \rightarrow N \backslash G$ provides a correspondence between the differential forms on $N \backslash G$ and the differential forms on $G$ that are invariant under the action of $N$. We can even strengthen this invariance condition.

Theorem 3.1 ([17, Theorem 1]). Let $\Omega^{*}(G)^{N}$ be the cochain complex of $N$-invariant differential forms on $G$ and let $\Omega^{*}(G)^{G}$ be the cochain complex of $G$-invariant differential forms on $G$. Then the inclusion $i: \Omega^{*}(G)^{G} \rightarrow \Omega^{*}(G)^{N}$ induces an isomorphism on cohomology.

As a conclusion so far, we have that

$$
H_{\text {triv }}^{*}(N, \mathbb{R}) \cong H_{d R}^{*}(N \backslash G) \cong H^{*}\left(\Omega^{*}(G)^{N}\right) \cong H^{*}\left(\Omega^{*}(G)^{G}\right) .
$$

Polynomial crystallographic action approach. Let $n$ be the Hirsch length of $N$. According to in [12 (see also [10]), $N$ admits a polynomial crystallographic action $\rho: N \rightarrow P\left(\mathbb{R}^{n}\right)$ of bounded degree. This $N$-action on $\mathbb{R}^{n}$ is free, so the quotient manifold $\rho(N) \backslash \mathbb{R}^{n}$ is again a compact $K(N, 1)$-manifold. We obtain an isomorphism

$$
H_{\text {triv }}^{*}(N, \mathbb{R}) \cong H_{d R}^{*}\left(\rho(N) \backslash \mathbb{R}^{n}\right) .
$$

Using the pullback of the projection mapping $p: \mathbb{R}^{n} \rightarrow \rho(N) \backslash \mathbb{R}^{n}$ one can show that differential forms on $\rho(N) \backslash \mathbb{R}^{n}$ correspond to $\rho(N)$-invariant differential forms on $\mathbb{R}^{n}$.

A diffeomorphism between $N \backslash G$ and $\rho(N) \backslash \mathbb{R}^{n}$. One of the major results in [13] is the fact that any affine crystallographic action of a $\mathcal{J}$-group $N$ can be extended to a simply transitive affine action of the Mal'cev completion $G$ of $N$ (this is in fact a translation of the contents of [13, Theorem 7.1]). This also holds for polynomial crystallographic actions.

Theorem 3.2. Let $N$ be a $\mathcal{J}$-group with Mal'cev completion $G$. Suppose $\rho: N \rightarrow$ $\mathrm{P}\left(\mathbb{R}^{n}\right)$ is a polynomial crystallographic action of bounded degree. Then there exists a unique simply transitive action $\bar{\rho}: G \rightarrow \mathrm{P}\left(\mathbb{R}^{n}\right)$, whose restriction to $N$ is $\rho$.

Proof. In [12, Theorem 3.3] (see also the example below) it is shown that $G$ admits a simply transitive polynomial action $\bar{\psi}: G \rightarrow \mathrm{P}\left(\mathbb{R}^{n}\right)$. Its restriction to $N$ yields a polynomial crystallographic action $\psi$ of $N$ on $\mathbb{R}^{n}$. The main result of 4 s states that there exists a polynomial map $p \in \mathrm{P}\left(\mathbb{R}^{n}\right)$ such that

$$
\rho(n)=p \circ \psi(n) \circ p^{-1} .
$$

Therefore the representation

$$
\bar{\rho}: G \rightarrow \mathrm{P}\left(\mathbb{R}^{n}\right): g \mapsto p \circ \bar{\psi}(g) \circ p^{-1}
$$


defines a simply transitive action of $G$ on $\mathbb{R}^{n}$ whose restriction to $N$ is just the action of $N$ on $\mathbb{R}^{n}$ given by $\rho$. This proves the existence of $\bar{\rho}$.

Analoguously we can prove uniqueness. Let $\bar{\rho}_{1}$ and $\bar{\rho}_{2}$ denote two simply transitive polynomial actions of $G$ on $\mathbb{R}^{n}$ whose restriction to $N$ is $\rho$. Let $A(H)$ be the algebraic closure construction from [4 for any subgroup $H \subseteq \mathrm{P}\left(\mathbb{R}^{n}\right)$ of bounded degree, and $U(H)$ the unipotent radical of such an $A(H)$. As $\rho(N) \subseteq \bar{\rho}_{1}(G)$, we have $A(\rho(N)) \subseteq A\left(\bar{\rho}_{1}(G)\right)$. Moreover, by [4, Lemma 5.1 and Remark 5.2], we also know that $A(\rho(N))=U(\rho(N))$ and $A\left(\bar{\rho}_{1}(G)\right)=U\left(\bar{\rho}_{1}(G)\right)=\bar{\rho}_{1}(G)$. Now both unipotent parts act simply transitively on $\mathbb{R}^{n}$, so $\bar{\rho}_{1}(G)=U(\rho(N))$. The same argument applied to $\bar{\rho}_{2}$ leads to the fact that $\bar{\rho}_{1}(G)=\bar{\rho}_{2}(G)$. Again we know there exists a polynomial map $p \in \mathrm{P}\left(\mathbb{R}^{n}\right)$ such that

$$
\forall g \in G: \bar{\rho}_{1}(g)=p \circ \bar{\rho}_{2}(g) \circ p^{-1} .
$$

This shows that conjugation with $p$ induces an automorphism of the group $\bar{\rho}_{1}(G)=$ $\bar{\rho}_{2}(G)$. This automorphism reduces to the identity on $\rho(N)$. As $\rho(N)$ is a uniform lattice of the nilpotent Lie group $\bar{\rho}_{1}(G)$, it has to be the identity on the whole group $\bar{\rho}_{1}(G)$, so $\bar{\rho}_{1}=\bar{\rho}_{2}$.

Example 3.1. Let $G$ be an $n$-dimensional, connected, simply connected and nilpotent Lie group, and $\mathcal{G}$ its Lie algebra. It is well known that in this setting the exponential map $\exp : \mathcal{G} \rightarrow G$ is a diffeomorphism. We will show that left multiplication with an element $g \in G$ corresponds to a polynomial diffeomorphism under exp. To this end, let $g=\exp (A), h=\exp (X) \in G$, where $X, Y \in \mathcal{G}$. Then

$$
g \cdot h=\exp (A) \cdot \exp (X)=\exp \left(A+X+\frac{1}{2}[A, X]+\ldots\right)
$$

by the Campbell-Baker-Hausdorff formula. As $\mathcal{G}$ is nilpotent, there are only finitely many nonzero terms. After choosing a basis for $\mathcal{G}$ and thus identifying $\mathcal{G}$ with $\mathbb{R}^{n}$, the expression

$$
A+X+\frac{1}{2}[A, X]+\ldots
$$

is actually a polynomial mapping $p_{A}(X): \mathbb{R}^{n} \rightarrow \mathbb{R}^{n}$ in the coordinates of $X$, with the coordinates of $A$ as parameters. It follows immediately from

$$
\exp \left(\left(p_{A} \circ p_{B}\right)(X)\right)=\exp (A) \cdot \exp (B) \cdot \exp (X)=\exp (C) \cdot \exp (X)=\exp \left(p_{C}(X)\right),
$$

where $\exp (C)=\exp (A) \cdot \exp (B)$, that $p_{A}(X)$ is a polynomial diffeomorphism for all $A$ and that the mapping

$$
m: G \rightarrow P\left(\mathbb{R}^{n}\right): g=\exp (A) \mapsto p_{A}
$$

is a group homomorphism. It is easy to see that the action of $G$ on $\mathbb{R}^{n}$ defined by $m$ is simply transitive.

Now consider a polynomial crystallographic action $\rho$ of bounded degree of a $\mathcal{J}$-group $N$ and let $\bar{\rho}$ be the unique extension to its Mal'cev completion $G$. The evaluation map Ev : $G \rightarrow \mathbb{R}^{n}$ defined by

$$
\operatorname{Ev}(g)=\bar{\rho}(g)(0)
$$


is a diffeomorphism, and for every $g \in G$, the following diagram commutes:

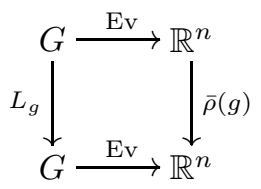

Of course, the map Ev induces a diffeomorphism between $N \backslash G$ and $\rho(N) \backslash \mathbb{R}^{n}$.

As the functor $\Omega^{*}(-)$ is contravariant, we can apply it to the diagram above to obtain the following corollary.

Corollary 3.3. $\Omega^{*}(\mathrm{Ev}): \Omega^{*}\left(\mathbb{R}^{n}\right)^{\rho(N)} \rightarrow \Omega^{*}(G)^{N}$ and $\Omega^{*}(\mathrm{Ev}): \Omega^{*}\left(\mathbb{R}^{n}\right)^{\bar{\rho}(G)} \rightarrow$ $\Omega^{*}(G)^{G}$ are isomorphisms of cochain complexes.

This allows us to formulate the analoguous version of Theorem 3.1 in the polynomial action approach.

Theorem 3.4. Let $\Omega^{*}\left(\mathbb{R}^{n}\right)^{\rho(N)}$ be the cochain complex of $\rho(N)$-invariant differential forms on $\mathbb{R}^{n}$ and let $\Omega^{*}\left(\mathbb{R}^{n}\right)^{\bar{\rho}(G)}$ be the cochain complex of $\bar{\rho}(G)$-invariant differential forms on $\mathbb{R}^{n}$. Then the inclusion $i: \Omega^{*}\left(\mathbb{R}^{n}\right)^{\bar{\rho}(G)} \rightarrow \Omega^{*}\left(\mathbb{R}^{n}\right)^{\rho(N)}$ induces an isomorphism on cohomology.

3.2. Polynomial cohomology for $\mathcal{J}$-groups. Computing the real cohomology of $N$ is now equivalent to computing the deRham cohomology of the $\bar{\rho}(G)$-invariant differential forms on $\mathbb{R}^{n}$. Recall that any differential $p$-form $\omega$ on $\mathbb{R}^{n}$ can be written as a sum

$$
\omega=\sum_{\sigma} \omega_{\sigma} d x_{\sigma(1)} \wedge d x_{\sigma(2)} \wedge \ldots \wedge d x_{\sigma(p)},
$$

where the sum ranges over the set $S(p, n-p)$ of all $(p, n-p)$-shuffles and each $\omega_{\sigma}$ is a differentiable map. A $(p, n-p)$-shuffle is a permutation $\sigma$ of $\{1,2, \ldots, n\}$ with

$$
\sigma(1)<\sigma(2)<\cdots<\sigma(p) \text { and } \sigma(p+1)<\sigma(p+2)<\cdots<\sigma(n) .
$$

It turns out that the $\bar{\rho}(G)$-invariant differential forms are polynomial.

Theorem 3.5. Let $G$ be a connected and simply connected nilpotent Lie group and let $\bar{\rho}: G \rightarrow P\left(\mathbb{R}^{n}\right)$ be a simply transitive polynomial action of $G$ on $\mathbb{R}^{n}$. Then every $\bar{\rho}(G)$-invariant differential form $\omega$ on $\mathbb{R}^{n}$ is polynomial, that is, if

$$
\omega=\sum_{\sigma \in S(p, n-p)} \omega_{\sigma} d x_{\sigma(1)} \wedge \ldots \wedge d x_{\sigma(p)},
$$

then $\omega_{\sigma}: \mathbb{R}^{n} \rightarrow \mathbb{R}$ is polynomial for every $\sigma \in S(p, n-p)$. Moreover, the complex $\Omega^{*}\left(\mathbb{R}^{n}\right)^{\bar{\rho}(G)}$ of $\bar{\rho}(G)$-invariant forms is finite dimensional, more precisely, $\operatorname{dim} \Omega^{p}\left(\mathbb{R}^{n}\right)^{\bar{\rho}(G)}=\left(\begin{array}{l}n \\ p\end{array}\right)$ for every $0 \leq p \leq n$.

Proof. Let $\omega \in \Omega^{p}\left(\mathbb{R}^{n}\right)$ be $\bar{\rho}(G)$-invariant.

Let $\mathcal{G}$ denote the Lie algebra of $G$. This Lie algebra can be identified with $\mathbb{R}^{n}$ by choosing a basis. As exp $: \mathcal{G} \rightarrow G$ is a diffeomorphism, it defines global coordinates on the manifold $G$. Clearly

$$
\omega=\left(\mathrm{Ev}^{-1}\right)^{*} \log ^{*} \exp ^{*} \operatorname{Ev}^{*}(\omega)=\left(\log \circ \operatorname{Ev}^{-1}\right)^{*} \exp ^{*}\left(\operatorname{Ev}^{*}(\omega)\right) .
$$


Lemma 2 in [4] shows that $\log \circ \mathrm{Ev}^{-1}$ is polynomial (recall we identified $\mathcal{G}$ with $\left.\mathbb{R}^{n}\right)$, so $\omega$ is polynomial iff $\exp ^{*}\left(\operatorname{Ev}^{*}(\omega)\right)$ is polynomial.

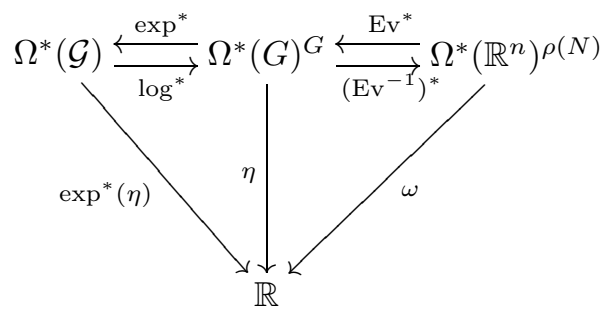

Let $\eta=\operatorname{Ev}^{*}(\omega) \in \Omega^{*}(G)^{G}$. The nilpotency of $G$ and the Campbell-BakerHausdorff formula imply that left multiplication inside $G$ is polynomial (of degree less than or equal to the nilpotency class of $G$ ) in the coordinates defined by exp. Now let $X \in \mathcal{G}$ and $\xi^{(1)}, \ldots, \xi^{(p)}$ be tangent vectors at $X$. Then

$$
\begin{aligned}
\exp ^{*}(\eta)(X)\left(\xi^{(1)}, \ldots, \xi^{(p)}\right) \\
\quad=\eta(\exp (X))\left(\exp _{*} \xi^{(1)}, \ldots, \exp _{*} \xi^{(p)}\right) \\
\quad=\eta(\exp (0))\left(\mathrm{L}_{\exp (-X)_{*}} \exp _{*} \xi^{(1)}, \ldots, \mathrm{L}_{\exp (-X)_{*}} \exp _{*} \xi^{(p)}\right) \\
\quad=\eta(\exp (0))\left(\exp _{*} \log _{*} \mathrm{~L}_{\exp (-X)_{*}} \exp _{*} \xi^{(1)}, \ldots, \exp _{*} \log _{*} \mathrm{~L}_{\exp (-X)_{*}} \exp _{*} \xi^{(p)}\right) \\
\quad=\exp ^{*}(\eta)(0)\left(\left(\log \circ \mathrm{L}_{\exp (-X)} \circ \exp \right)_{*} \xi^{(1)}, \ldots,\left(\log \circ \mathrm{L}_{\exp (-X)} \circ \exp \right)_{*} \xi^{(p)}\right)
\end{aligned}
$$

This last expression is polynomial in the coordinates of $X \in \mathcal{G}$, so $\eta$ is polynomial in the coordinates defined by exp, and $\omega=\left(\mathrm{Ev}^{*}\right)^{-1}(\eta)$ is also.

As the $\bar{\rho}(G)$-action on $\mathbb{R}^{n}$ is simply transitive, it is easy to see that any $\bar{\rho}(G)$ invariant form $\omega$ is determined by its value in the origin. But this means $\Omega^{p}\left(\mathbb{R}^{n}\right)^{\bar{\rho}(G)}$ corresponds to the vector space of alternating $p$-forms on the tangent space at the origin, so $\Omega^{p}\left(\mathbb{R}^{n}\right)^{\bar{\rho}(G)}$ has dimension $\left(\begin{array}{l}n \\ p\end{array}\right)$.

Once we have restricted ourselves to the polynomial differential forms, $\rho(N)$ invariance implies $\bar{\rho}(G)$-invariance.

Proposition 3.6. Let $N$ be a J -group with Mal'cev completion $G, \rho: N \rightarrow \mathrm{P}\left(\mathbb{R}^{n}\right)$ a crystallographic action and $\bar{\rho}: G \rightarrow \mathrm{P}\left(\mathbb{R}^{n}\right)$ its extension to $G$. Then every $\rho(N)$ invariant polynomial differential form $\omega \in \Omega^{*}\left(\mathbb{R}^{n}\right)$ is also $\bar{\rho}(G)$-invariant.

The proof of this proposition uses the following well-known lemma.

Lemma 3.7. Let $n, k \in \mathbb{N}_{0}$ and $n \geq k$. Suppose $\Gamma \subseteq \mathbb{R}^{k}$ is a generating set for $\mathbb{R}^{k}$ as a vector space and $p \in \mathbb{R}\left[x_{1}, \ldots, x_{k}, x_{k+1}, \ldots, x_{n}\right]$ is a polynomial. If

$$
p\left(x_{1}+\gamma_{1}, \ldots, x_{k}+\gamma_{k}, x_{k+1}, \ldots, x_{n}\right)=p\left(x_{1}, \ldots, x_{k}, x_{k+1}, \ldots, x_{n}\right)
$$

for every $\left(\gamma_{1}, \ldots, \gamma_{k}\right) \in \Gamma$ and every $\left(x_{1}, \ldots, x_{k}, x_{k+1}, \ldots, x_{n}\right) \in \mathbb{R}^{n}$, then $p$ does not depend on the variables $x_{1}, \ldots, x_{k}$.

Proof of Proposition 3.6. It follows from [4 that every polynomial structure $\bar{\rho}$ : $G \rightarrow P\left(\mathbb{R}^{n}\right)$ is polynomially conjugated to $m: G \rightarrow P\left(\mathbb{R}^{n}\right)$, where $m$ is the polynomial crystallographic action obtained by expressing left multiplication in $G$ in Mal'cev coordinates of the second kind (see Example 3.1 or again [4]). So there is 
a $q \in P\left(\mathbb{R}^{n}\right)$ such that

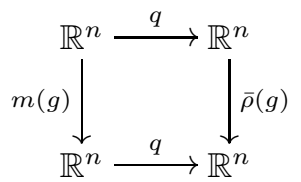

for every $g \in G$. By applying the functor $\Omega^{*}(-)$ to the commutative diagram above we see that the action of $\bar{\rho}(g)^{*}$ on differential forms of $\mathbb{R}^{n}$ corresponds to the action of $m(g)^{*}$. This implies that it is sufficient to show that a polynomial $m(N)$-invariant differential form on $\mathbb{R}^{n}$ is also $m(G)$-invariant.

Using the coordinates for $G$ defined by the diffeomorphism exp : $\mathbb{R}^{n} \cong \mathcal{G} \rightarrow G$ we see that for $Y=\left(y_{1}, y_{2}, \ldots, y_{n}\right) \in \mathcal{G}, m(\exp (Y))$ is also polynomial in the $y_{i}$. This makes the invariance condition for a polynomial differential form $\omega$ on $\mathbb{R}^{n}$,

$$
\left(m(\exp (Y))^{*} \omega\right)\left(x_{1}, x_{2}, \ldots, x_{n}\right)=\omega\left(x_{1}, x_{2}, \ldots, x_{n}\right),
$$

entirely polynomial: we get a set of equations that are polynomial in the $x_{i}$ and in the $y_{j}$. Now as $N$ is a lattice in $G, \log (N)$ is a generating set for $\mathcal{G}$. Given the equations (3.1) are satisfied for all $Y \in \log (N)$, the lemma assures that these equations are true for all $Y \in \mathcal{G}$. But this means $m(N)$-invariance implies $m(G)$ invariance.

Theorem 3.8. Let $N$ be a $\mathcal{J}$-group and $\rho: N \rightarrow P\left(\mathbb{R}^{n}\right)$ a polynomial crystallographic action of bounded degree. Let $\Omega_{P}^{*}\left(\mathbb{R}^{n}\right)^{\rho(N)}$ be the cochain complex of $\rho(N)$ invariant polynomial differential forms on $\mathbb{R}^{n}$. Then there is an isomorphism of cohomology spaces

$$
H^{*}(N, \mathbb{R}) \cong H^{*}\left(\Omega_{P}^{*}\left(\mathbb{R}^{n}\right)^{\rho(N)}\right)
$$

under which the ring structures correspond.

Once we know we can restrict to polynomial differential forms, the invariance condition is a very computational one. Using Lemma 3.7, one can actually compute the invariant differential forms.

Example 3.2. Let us consider the case $N=\mathbb{Z}^{n}$ for some $n \in \mathbb{N}_{0}$. The translation action $\rho: \mathbb{Z}^{n} \rightarrow \operatorname{Aff}\left(\mathbb{R}^{n}\right)$ yields a free and properly discontinuous action, the quotient space is the $n$-torus. Now let $\left(e_{1}, \ldots, e_{n}\right)$ be the standard basis of $\mathbb{Z}^{n}$ and $\omega \in \Omega_{P}^{p}\left(\mathbb{R}^{n}\right)$. Then the invariance condition states that

$$
(\omega)\left(x-e_{i}\right)\left(\xi^{(1)}, \ldots, \xi^{(p)}\right)=\omega(x)\left(\xi^{(1)}, \ldots, \xi^{(p)}\right)
$$

or, written in full,

$$
\sum_{\sigma \in S(p, n-p)} \omega_{\sigma}\left(x-e_{i}\right) d x_{\sigma(1)} \wedge \ldots \wedge d x_{\sigma(p)}=\sum_{\sigma \in \mathcal{S}(p, n-p)} \omega_{\sigma}(x) d x_{\sigma(1)} \wedge \ldots \wedge d x_{\sigma(p)} .
$$

Again, $\mathcal{S}(p, n-p)$ denotes the set of all $(p, n-p)$-shuffles. Lemma 3.7 then shows that every $\omega_{\sigma}$ is independent of $x_{i}$, for every $i \in\{1, \ldots, n\}$. So all functions $\omega_{\sigma}$ are in fact constants, and subsequently the differential on any $\rho\left(\mathbb{Z}^{n}\right)$-invariant form is zero. We thus obtain the well-known result that $H^{*}\left(\mathbb{Z}^{n}, \mathbb{R}\right) \cong \mathrm{Alt}{ }^{*}\left(\mathbb{R}^{n}\right)$, the graded $\mathbb{R}$-algebra of alternating forms on $\mathbb{R}^{n}$.

Example 3.3. Consider the generalized Heisenberg group

$$
H_{k}=\left\langle a, b, c \|[b, a]=c^{k},[b, c]=[a, c]=1\right\rangle,
$$


where $k \in \mathbb{N}_{0}$. This group has an affine crystallographic action $\rho: N \rightarrow \operatorname{Aff}\left(\mathbb{R}^{3}\right)$ given by

$$
\rho(a)=\left(\begin{array}{cccc}
1 & 0 & -\frac{k}{2} & 0 \\
0 & 1 & 0 & 1 \\
0 & 0 & 1 & 0 \\
0 & 0 & 0 & 1
\end{array}\right), \quad \rho(b)=\left(\begin{array}{cccc}
1 & \frac{k}{2} & 0 & 0 \\
0 & 1 & 0 & 0 \\
0 & 0 & 1 & 1 \\
0 & 0 & 0 & 1
\end{array}\right), \quad \rho(c)=\left(\begin{array}{cccc}
1 & 0 & 0 & 1 \\
0 & 1 & 0 & 0 \\
0 & 0 & 1 & 0 \\
0 & 0 & 0 & 1
\end{array}\right)
$$

(see [9, p. 159]). We would like to compute the $\rho\left(H_{k}\right)$-invariant polynomial differential forms $\omega$ on $\mathbb{R}^{3}$. The invariance condition is $\rho(n)^{*}(\omega)=\omega$, or explicitly,

$$
\omega(\rho(n)(x))\left(\xi^{(1)}, \ldots, \xi^{(p)}\right)=\omega(x)\left(\rho\left(n^{-1}\right)_{*} \xi^{(1)}, \ldots, \rho\left(n^{-1}\right)_{*} \xi^{(p)}\right)
$$

for all $n \in H_{k}$, all $x \in \mathbb{R}^{3}$ and all vectors $\xi^{(1)}, \xi^{(2)}, \ldots, \xi^{(p)}$ tangent to $\mathbb{R}^{3}$ at $\rho(n)(x)$. Of course it is sufficient to check the invariance of the differential forms under the action of the generators.

Computing 0-forms. Suppose $f \in \Omega^{0}\left(\mathbb{R}^{3}\right)$. The invariance conditions are

$$
\begin{aligned}
f\left(x_{1}-\frac{k}{2} x_{3}, x_{2}+1, x_{3}\right) & =f\left(x_{1}, x_{2}, x_{3}\right), \\
f\left(x_{1}+\frac{k}{2} x_{2}, x_{2}, x_{3}+1\right) & =f\left(x_{1}, x_{2}, x_{3}\right), \\
f\left(x_{1}+1, x_{2}, x_{3}\right) & =f\left(x_{1}, x_{2}, x_{3}\right) .
\end{aligned}
$$

Using Lemma 3.7, we deduce from condition (3.4) that $f$ does not depend on $x_{1}$. Once we know this, conditions (3.2) and (3.3) imply analogously that $f$ is a constant mapping from $\mathbb{R}^{3}$ to $\mathbb{R}$. As the differential of a constant mapping is zero, we obtain that

$$
H_{\text {triv }}^{0}\left(H_{k}, \mathbb{R}\right) \cong \mathbb{R}
$$

Computing 1-forms. Let $\omega=\omega_{1} d x_{1}+\omega_{2} d x_{2}+\omega_{3} d x_{3} \in \Omega^{1}\left(\mathbb{R}^{3}\right)$. The invariance conditions are

$$
\sum_{i=1}^{3} \omega_{i}\left(x_{1}-\frac{k}{2} x_{3}, x_{2}+1, x_{3}\right) d x_{i}=\omega_{1}(x) d x_{1}+\omega_{2}(x) d x_{2}+\left(\omega_{3}(x)-\frac{k}{2} \omega_{1}(x)\right) d x_{3},
$$

$$
\begin{aligned}
& \sum_{i=1}^{3} \omega_{i}\left(x_{1}+\frac{k}{2} x_{2}, x_{2}, x_{3}+1\right) d x_{i}=\omega_{1}(x) d x_{1}+\left(\omega_{2}(x)+\frac{k}{2} \omega_{1}(x)\right) d x_{2}+\omega_{3}(x) d x_{3}, \\
& \text { (3.7) } \sum_{i=1}^{3} \omega_{i}\left(x_{1}+1, x_{2}, x_{3}\right) d x_{i}=\sum_{i=1}^{3} \omega_{i}(x) d x_{i} .
\end{aligned}
$$

It follows from vector equation (3.7) that $\omega_{1}, \omega_{2}$ and $\omega_{3}$ are independent of $x_{1}$. Equation (3.6) is in fact a set of three equations,

$$
\left\{\begin{array}{l}
\omega_{1}\left(x_{2}, x_{3}+1\right)=\omega_{1}\left(x_{2}, x_{3}\right) \\
\omega_{2}\left(x_{2}, x_{3}+1\right)=\omega_{2}\left(x_{2}, x_{3}\right)+\frac{k}{2} \omega_{1}\left(x_{2}, x_{3}\right), \\
\omega_{3}\left(x_{2}, x_{3}+1\right)=\omega_{3}\left(x_{2}, x_{3}\right)
\end{array}\right.
$$

implying that $\omega_{1}$ and $\omega_{3}$ are independent of $x_{3}$ and that $\omega_{2}\left(x_{2}, x_{3}\right)=$ $c_{2}\left(x_{2}\right)+x_{3} \frac{k}{2} \omega_{1}\left(x_{2}\right)$. Analoguously (3.5) implies that $\omega_{1}$ and $\omega_{2}$ do not depend on $x_{2}$ (so $\omega_{1}=c_{1}$ and $\omega_{2}\left(x_{3}\right)=c_{2}+x_{3} \frac{k}{2} c_{1}$, where $c_{1}$ and $c_{2}$ are 
real constants) and that $\omega_{3}\left(x_{2}\right)=c_{3}+x_{2} \frac{k}{2} c_{1}$ for $c_{3}$ a real constant. This leads us to conclude that

$$
\omega\left(x_{1}, x_{2}, x_{3}\right)=c_{1} d x_{1}+\left(c_{2}-\frac{k}{2} c_{1} x_{3}\right) d x_{2}+\left(c_{3}+\frac{k}{2} c_{1} x_{2}\right) d x_{3} .
$$

The differential of such a 1 -form is

$$
d \omega\left(x_{1}, x_{2}, x_{3}\right)=k c_{1} d x_{2} \wedge d x_{3}
$$

indicating that

$$
H_{\text {triv }}^{1}\left(H_{k}, \mathbb{R}\right) \cong \mathbb{R}^{2} .
$$

Computing 2-forms. These computations are completely analoguous to the case of the 1-forms, and we only write the result. A polynomial $\rho\left(H_{k}\right)$ invariant 2 -form can be written as

$\omega\left(x_{1}, x_{2}, x_{3}\right)=c_{1,2} d x_{1} \wedge d x_{2}+c_{1,3} d x_{1} \wedge d x_{3}+\left(c_{2,3}-\frac{k}{2} c_{1,2} x_{3}-\frac{k}{2} c_{1,3} x_{2}\right) d x_{2} \wedge d x_{3}$, where the $c_{i, j}$ are real constants. This form has a zero differential, so we see that

$$
H_{\text {triv }}^{2}\left(H_{k}, \mathbb{R}\right) \cong \mathbb{R}^{2}
$$

Computing 3-forms. This last case is similar to the 0 -forms case. A polynomial $\rho\left(H_{k}\right)$-invariant 3 -form looks like

$$
\omega\left(x_{1}, x_{2}, x_{3}\right)=c_{1,2,3} d x_{1} \wedge d x_{2} \wedge d x_{3}
$$

with $c_{1,2,3}$ a real constant. This form trivially has a zero differential, so

$$
H_{\text {triv }}^{3}\left(H_{k}, \mathbb{R}\right) \cong \mathbb{R} \text {. }
$$

Of course, the above example could also be computed using other means (e.g. Poincaré duality). However, exactly the same technique can be used to obtain the results below, which are less obvious to compute.

Example 3.4. The real cohomology of the 8-dimensional, 4-step nilpotent group

$$
\begin{array}{llll}
N=\left\langle e_{1}, e_{2}, e_{3}, e_{4}, e_{5}, e_{6}, e_{7}, e_{8} \|\right. & {\left[e_{1}, e_{2}\right]=e_{4}^{2},} & {\left[e_{2}, e_{3}\right]=e_{5}^{2},} \\
& {\left[e_{1}, e_{5}\right]=e_{6}^{-1} e_{8}^{-2},} & {\left[e_{3}, e_{4}\right]=e_{6},} \\
& {\left[e_{2}, e_{5}\right]=e_{7} e_{8}^{-2},} & {\left[e_{2}, e_{6}\right]=e_{8}^{-2},} \\
& {\left[e_{4}, e_{5}\right]=e_{8},} & \left.\left[e_{1}, e_{7}\right]=e_{8}^{4}\right\rangle
\end{array}
$$

is given by

$$
\begin{array}{lll}
H_{\text {triv }}^{0}(N, \mathbb{R}) \cong \mathbb{R}, & H_{\text {triv }}^{1}(N, \mathbb{R}) \cong \mathbb{R}^{3}, & H_{\text {triv }}^{2}(N, \mathbb{R}) \cong \mathbb{R}^{6}, \\
H_{\text {triv }}^{3}(N, \mathbb{R}) \cong \mathbb{R}^{9}, & H_{\text {triv }}^{4}(N, \mathbb{R}) \cong \mathbb{R}^{10}, & H_{\text {triv }}^{5}(N, \mathbb{R}) \cong \mathbb{R}^{9}, \\
H_{\text {triv }}^{6}(N, \mathbb{R}) \cong \mathbb{R}^{6}, \quad H_{\text {triv }}^{7}(N, \mathbb{R}) \cong \mathbb{R}^{3}, & H_{\text {triv }}^{8}(N, \mathbb{R}) \cong \mathbb{R} .
\end{array}
$$

Example 3.5. The real cohomology of the free 4-generated 2-step nilpotent group

$$
\begin{aligned}
& F_{2,4}=\left\langle e_{1}, e_{2}, e_{3}, e_{4}, e_{5}, e_{6}, e_{7}, e_{8}, e_{9}, e_{10} \quad \| \quad\left[e_{1}, e_{2}\right]=e_{5}, \quad\left[e_{1}, e_{3}\right]=e_{6},\right. \\
& {\left[e_{1}, e_{4}\right]=e_{7}, \quad\left[e_{2}, e_{3}\right]=e_{8},} \\
& \left.\left[e_{2}, e_{4}\right]=e_{9}, \quad\left[e_{3}, e_{4}\right]=e_{10}, \quad\right\rangle
\end{aligned}
$$

is given by

$$
\begin{array}{lll}
H_{\text {triv }}^{0}(G, \mathbb{R}) \cong \mathbb{R}, & H_{\text {triv }}^{1}(G, \mathbb{R}) \cong \mathbb{R}^{4}, \quad H_{\text {triv }}^{2}(G, \mathbb{R}) \cong \mathbb{R}^{20}, \\
H_{\text {triv }}^{3}(G, \mathbb{R}) \cong \mathbb{R}^{56}, \quad H_{\text {triv }}^{4}(G, \mathbb{R}) \cong \mathbb{R}^{84}, \quad H_{\text {triv }}^{5}(G, \mathbb{R}) \cong \mathbb{R}^{90}, \\
H_{\text {triv }}^{6}(G, \mathbb{R}) \cong \mathbb{R}^{84}, \quad H_{\text {triv }}^{7}(G, \mathbb{R}) \cong \mathbb{R}^{56}, \quad H_{\text {triv }}^{8}(G, \mathbb{R}) \cong \mathbb{R}^{20}, \\
H_{\text {triv }}^{9}(G, \mathbb{R}) \cong \mathbb{R}^{4}, \quad H_{\text {triv }}^{10}(G, \mathbb{R}) \cong \mathbb{R}^{\mathrm{R}} .
\end{array}
$$




\section{Cohomology of Virtually Nilpotent Groups}

In this section, we will extend the cohomology theory for $\mathcal{J}$-groups developed above to the class of virtually $\mathcal{J}$-groups. It is well known (e.g. see [19]) that this is in fact the class of finitely generated virtually nilpotent groups.

4.1. Cohomology of virtually $\mathcal{J}$-groups. We can relate the cohomology of a finite extension $E$ of a group $N$ immediately to the cohomology of $N$, provided the coefficient module is appropriate.

Proposition 4.1. Let $E$ be a group, let $N$ be a normal subgroup of finite index and let $F=E / N$. Suppose the E-module $A$ is a vector space uniquely divisible by the index of $F$. Then

$$
H^{*}(E, A) \cong H^{*}(N, A)^{F}
$$

Proof. The Hochschild-Serre spectral sequence ([14, [6]) states that

$$
H^{p}\left(F, H^{q}(N, A)\right) \Rightarrow H^{p+q}(E, A) .
$$

The $F$-module structure of $H^{q}(N, A)$ is determined by conjugation inside $E$ and the $E$-module structure of $A$. Since $F$ is finite, the composition of the restriction map and corestriction map

$$
\operatorname{cor}_{1, F}^{*} \circ \operatorname{res}_{F, 1}^{*}: H^{*}\left(F, H^{q}(N, A)\right) \rightarrow H^{*}\left(1, H^{q}(N, A)\right) \rightarrow H^{*}\left(F, H^{q}(N, A)\right)
$$

is just multiplication by the index of $F$ (see [6, Proposition 9.5]), which is an isomorphism of $H^{*}\left(F, H^{q}(N, A)\right)$. But $H^{p}\left(1, H^{q}(N, A)\right)=0$ when $p \geq 1$ so $H^{p}\left(F, H^{q}(N, A)\right)$ is trivial as well when $p \neq 0$. But this means that the HochschildSerre sectral sequence reduces to its $E_{0, q}^{2}$-terms, and that

$$
H^{q}(E, A) \cong H^{0}\left(F, H^{q}(N, A)\right) \cong H^{q}(N, A)^{F} .
$$

Let $E$ be a virtually nilpotent group of Hirsch length $n$, let $N$ be a normal $\mathcal{J}$ subgroup of finite index with a polynomial representation $\rho: N \rightarrow P\left(\mathbb{R}^{n}\right)$ and let $F=E / N$. To assure that the conclusion of section 2 applies, we suppose that the $E$-module structure of $\mathbb{R}$ is given by a group homomorphism $\varphi: E \rightarrow \operatorname{Aut}(\mathbb{R})$ such that $\varphi(N)=\{1\}$. The induced morphism from $E / N$ to $\operatorname{Aut}(\mathbb{R})$ will also be denoted by $\varphi$. The proposition above with $A=\mathbb{R}$ yields

$$
H_{\varphi}^{*}(E, \mathbb{R}) \cong H_{\text {triv }}^{*}(N, \mathbb{R})^{F} \cong H_{d R}^{*}\left(\rho(N) \backslash \mathbb{R}^{n}\right)^{F} .
$$

This observation allows us to generalize the results of the cohomology of $\mathcal{J}$-groups to analoguous results on the cohomology of virtually $\mathcal{J}$-groups. To obtain these, we need to trace the $F$-action involved throughout the isomorphisms explained in section 2 .

Following the account given by [6], we use the action of $N$ on $\mathbb{R}^{n}$ given by $\rho$ to consider the chain complex $C_{*}\left(\mathbb{R}^{n}\right)$ as a complete resolution of $\mathbb{Z}$ as a trivial $\mathbb{Z} N$ module. To define the $F$-action on $H_{\text {triv }}^{*}(N, \mathbb{R})$ we extend the $N$-module structure of $C_{*}\left(\mathbb{R}^{n}\right)$ to an $E$-module structure. This is done by extending the action of $N$ on $\mathbb{R}^{n}$.

Proposition 4.2. Let $\rho: N \rightarrow P\left(\mathbb{R}^{n}\right)$ be a polynomial crystallographic action. Then $\rho$ can be extended to a group homomorphism $\tilde{\rho}: E \rightarrow P\left(\mathbb{R}^{n}\right)$. 
Proof. In [12] it was shown that $E$ admits a polynomial crystallographic action $\rho^{\prime}$ of bounded degree on $\mathbb{R}^{n}$. As the restriction of this action $\rho^{\prime}$ to $N$ is polynomially conjugated to the action $\rho$ of $N$ (by the main result of [4 again), we obtain that this polynomial conjugate of $\rho^{\prime}$ can serve as an extension of the action $\rho$ to the group $E$.

Remark. At this point, one could ask whether the conclusion of section 2 applies to this more general setting of virtually nilpotent groups using this extended group action. The quotient space, however, is not necessarily a $K(E, 1)$-space because the virtually nilpotent group $E$ can have nontrivial torsion. This makes it impossible for $E$ to act freely.

Using the extension $\tilde{\rho}: E \rightarrow P\left(\mathbb{R}^{n}\right), C_{*}\left(\mathbb{R}^{n}\right)$ becomes a complex of $E$-modules. The lifting procedure presented in [6, p. 79] eventually describes the action of an $e \in E$ on a cocycle $f \in \operatorname{Hom}_{\mathbb{Z} N}\left(C_{*}\left(\mathbb{R}^{n}\right), \mathbb{R}\right)$ as

$$
{ }^{e} f=\varphi(e) f \tilde{\rho}\left(e^{-1}\right)_{*} .
$$

As $f$ is a $\mathbb{Z} N$-homomorphism, this actually defines an $F$-action on $H^{*}(N, \mathbb{R})$.

The $F$-action on $H_{\text {sing }}^{*}\left(\rho(N) \backslash \mathbb{R}^{n}, \mathbb{R}\right)$. We would like to describe the $F$-action on the complex $\operatorname{Hom}_{\mathbb{Z}}\left(C_{*}\left(\rho(N) \backslash \mathbb{R}^{n}\right), \mathbb{R}\right)$ induced by the $F$-action on the complex $\operatorname{Hom}_{\mathbb{Z} N}\left(C_{*}\left(\mathbb{R}^{n}\right), \mathbb{R}\right)$ as defined above:

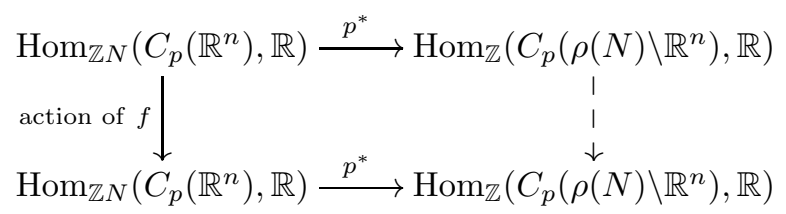

First, observe that the $E$-action on $\mathbb{R}^{n}$ induces an action of $E$ on $\rho(N) \backslash \mathbb{R}^{n}$ defined by

$$
{ }^{e} y=p\left({ }^{e} x\right)=p(\tilde{\rho}(e)(x)),
$$

where $p: \mathbb{R}^{n} \rightarrow \rho(N) \backslash \mathbb{R}^{n}$ is the projection, $y \in \rho(N) \backslash \mathbb{R}^{n}, x \in p^{-1}(y)$ and $e \in E$. In this way we obtain a group homomorphism $\psi: E \rightarrow \mathcal{D}\left(\rho(N) \backslash \mathbb{R}^{n}\right)$ such that $\psi(N)=1$. This allows us to factor out $N$ and consider $\psi$ as a group homomorphism from $F$ to $\mathcal{D}\left(\rho(N) \backslash \mathbb{R}^{n}\right)$.

As the isomorphism between $H^{*}(N, \mathbb{R})$ and $H_{\text {sing }}^{*}\left(\rho(N) \backslash \mathbb{R}^{n}, \mathbb{R}\right)$ is given by $p^{*}$, we see that

$$
{ }^{f} p^{*}(u)=\varphi(f) \circ u \circ p_{*} \circ \tilde{\rho}\left(s(f)^{-1}\right)_{*}=\varphi(f) \circ u \circ \psi\left(f^{-1}\right)_{*} \circ p_{*}
$$

for $u \in \operatorname{Hom}_{\mathbb{Z}}\left(C_{*}\left(\rho(N) \backslash \mathbb{R}^{n}\right), \mathbb{R}\right)$ and $f \in F$. This determines the $F$-action on $H_{\text {sing }}^{*}\left(\rho(N) \backslash \mathbb{R}^{n}, \mathbb{R}\right)$ : explicitly, for a $u \in \operatorname{Hom}_{\mathbb{Z}}\left(C_{*}\left(\rho(N) \backslash \mathbb{R}^{n}\right), \mathbb{R}\right)$ we have that

$$
{ }^{f} u=\varphi(f) \circ u \circ \psi\left(f^{-1}\right)^{*},
$$

where $f \in F$. 
The $F$-action on $H_{d R}^{*}\left(\rho(N) \backslash \mathbb{R}^{n}\right)$. Let $\omega \in \Omega^{p}\left(\rho(N) \backslash \mathbb{R}^{n}\right)$ and $f \in F$. If $T:[0,1]^{p} \rightarrow$ $\rho(N) \backslash \mathbb{R}^{n}$ is a differential singular $p$-cube, then

$$
{ }^{f} \Phi(\omega)(T)=\varphi(f) \int_{\psi\left(f^{-1}\right)_{*}(T)} \omega .
$$

But $\left(\psi\left(f^{-1}\right)_{*} T\right)^{*} \omega=\left(\psi\left(f^{-1}\right) \circ T\right)^{*} \omega=T^{*}\left(\psi\left(f^{-1}\right)^{*} \omega\right)$, so

$$
\int_{\psi\left(f^{-1}\right)_{*}(T)} \omega=\int_{T} \psi\left(f^{-1}\right)^{*} \omega
$$

which leads us to conclude that

$$
{ }^{f} \omega=\varphi(f) \psi\left(f^{-1}\right)^{*} \omega .
$$

With this action $H_{d R}^{*}\left(\rho(N) \backslash \mathbb{R}^{n}\right)^{F}$ is the cohomology of differential forms $\omega$ on $\rho(N) \backslash \mathbb{R}^{n}$ that satisfy

$$
\varphi(f) \omega\left(\psi\left(f^{-1}\right)(y)\right)\left(\psi\left(f^{-1}\right)_{*} \xi^{(1)}, \ldots, \psi\left(f^{-1}\right)_{*} \xi^{(p)}\right)=\omega(y)\left(\xi^{(1)}, \ldots, \xi^{(p)}\right)
$$

for every $f \in F, y \in \rho(N) \backslash \mathbb{R}^{n}$ and tangent vectors $\xi^{(1)}, \ldots, \xi^{(p)}$ to $\rho(N) \backslash \mathbb{R}^{n}$ at $y$. This is, again, the invariance condition for differential forms, but now these forms live on $\rho(N) \backslash \mathbb{R}^{n}$.

The $F$-action on $H_{d R}^{*}\left(\Omega^{*}\left(\mathbb{R}^{n}\right)^{\rho(N)}\right)$. Let us transfer the $F$-invariant forms on $\rho(N) \backslash \mathbb{R}^{n}$ to $\mathbb{R}^{n}$. First of all, define an $E$-action on $\Omega^{p}\left(\mathbb{R}^{n}\right)$ by

$$
{ }^{e} \eta(x)\left(\xi^{(1)}, \ldots, \xi^{(p)}\right)=\varphi(e) \eta\left(\tilde{\rho}\left(e^{-1}\right)(x)\right)\left(\tilde{\rho}\left(e^{-1}\right)_{*} \xi^{(1)}, \ldots, \tilde{\rho}\left(e^{-1}\right)_{*} \xi^{(p)}\right) .
$$

This action induces an $F$-action when restricted to the $\rho(N)$-invariant forms on $\mathbb{R}^{n}$. Now let $\omega \in \Omega^{p}\left(\rho(N) \backslash \mathbb{R}^{n}\right)^{F}$ be a differential form on $\rho(N) \backslash \mathbb{R}^{n}$ such that the invariance condition (4.1) is satisfied for all $f \in F$. Using the pullback of the projection $p: \mathbb{R}^{n} \rightarrow \rho(N) \backslash \mathbb{R}^{n}$ we obtain a form $p^{*}(\omega) \in \Omega^{p}\left(\mathbb{R}^{n}\right)^{\rho(N)}$. Now the $F$-invariance of $\omega$ yields

$$
\begin{aligned}
{ }^{e} p^{*} & (\omega)(x)\left(\xi^{(1)}, \xi^{(2)}, \ldots, \xi^{(p)}\right) \\
& =\varphi(e) \omega\left(p \circ \tilde{\rho}\left(e^{-1}\right)(x)\right)\left(p_{*} \tilde{\rho}\left(e^{-1}\right)_{*} \xi^{(1)}, \ldots, p_{*} \tilde{\rho}\left(e^{-1}\right)_{*} \xi^{(p)}\right) \\
& =\varphi(f) \omega\left(\psi\left(f^{-1}\right)(p(x))\right)\left(\psi\left(f^{-1}\right)_{*} p_{*} \xi^{(1)}, \ldots, \psi\left(f^{-1}\right)_{*} p_{*} \xi^{(p)}\right) \\
& =\omega(p(x))\left(p_{*} \xi^{(1)}, \ldots, p_{*} \xi^{(p)}\right) \\
& =p^{*}(\omega)(x)\left(\xi^{(1)}, \ldots, \xi^{(p)}\right),
\end{aligned}
$$

where $e \in E$ and $e N=f$ for $F=E / N$. So $p^{*}(\omega)$ is actually a $\tilde{\rho}(E)$-invariant form on $\mathbb{R}^{n}$, that is, a differential form $\omega$ on $\mathbb{R}^{n}$ that satisfies

$$
\varphi(e) \omega\left(\tilde{\rho}\left(e^{-1}(x)\right)\left(\tilde{\rho}\left(e^{-1}\right)_{*} \xi^{(1)}, \ldots, \tilde{\rho}\left(f^{-1}\right)_{*} \xi^{(p)}\right)=\omega(x)\left(\xi^{(1)}, \ldots, \xi^{(p)}\right)\right.
$$

for every $e \in E, x \in \mathbb{R}^{n}$ and tangent vectors $\xi^{(1)}, \ldots, \xi^{(p)}$ to $\mathbb{R}^{n}$ at $x$. Analoguously one can show that every $\tilde{\rho}(E)$-invariant form on $\mathbb{R}^{n}$ corresponds to an $F$-invariant form on $\rho(N) \backslash \mathbb{R}^{n}$.

Theorem 4.3. Let $E$ be a virtually nilpotent group of Hirsch length $n$ and let $N$ be a normal $\mathcal{J}$-subgroup of finite index. Let $\tilde{\rho}: E \rightarrow P\left(\mathbb{R}^{n}\right)$ be a group homomorphism such that the action of $N$ defined by $\rho=\left.\tilde{\rho}\right|_{N}$ is a polynomial crystallographic action. Suppose the morphism $\varphi: E \rightarrow \operatorname{Aut}(\mathbb{R})$ defines an E-module structure on $\mathbb{R}$ such 
that $\varphi(N)=\{1\}$. Let $\Omega_{P}^{*}\left(\mathbb{R}^{n}\right)^{\tilde{\rho}(E), \varphi(E)}$ be the cochain complex of the polynomial differential forms on $\mathbb{R}^{n}$ satisfying the invariance condition (4.2) for $E$. Then

$$
H_{\varphi}^{*}(E, \mathbb{R}) \cong H^{*}\left(\Omega_{P}^{*}\left(\mathbb{R}^{n}\right)^{\tilde{\rho}(E), \varphi(E)}\right) .
$$

Moreover, the product structures of both cohomology spaces correspond under this isomorphism.

This theorem is clear once we have established the following fact.

Lemma 4.4. Let $F$ be a finite group acting on a vector space $V$ over a field that is uniquely divisible by the order of $F$. If $W$ is an $F$-invariant subspace of $V$, then

$$
V^{F} / W^{F} \cong(V / W)^{F} \text {. }
$$

Proof of Lemma 4.4. The short exact sequence $0 \rightarrow W \rightarrow V \rightarrow V / W \rightarrow 0$ of $F$-modules induces a long exact sequence of cohomology groups

$$
0 \rightarrow H^{0}(F, W) \rightarrow H^{0}(F, V) \rightarrow H^{0}(F, V / W) \rightarrow H^{1}(F, W) \rightarrow \cdots .
$$

As $H^{1}(F, W)=0$ (since $W$ is uniquely divisible by the order of $F$ ), the sequence above induces the short exact seqence

$$
0 \rightarrow H^{0}(F, W) \rightarrow H^{0}(F, V) \rightarrow H^{0}(F, V / W) \rightarrow 0
$$

which is exactly what we had to show.

Example 3.3 continued. Let us have a look at the finite extension $E$ of the generalised Heisenberg group given by

$$
\begin{array}{cll}
E=\langle a, b, c, \alpha \| & {[b, a]=c^{k},} & \alpha c=c \alpha, \\
& {[c, a]=1,} & {[c, b]=1,} \\
& \alpha a=a^{-1} \alpha c^{l}, \quad \alpha b=b^{-1} \alpha c^{m}, \\
& \alpha^{2}=c^{n}
\end{array}
$$

for $k, l, m, n \in \mathbb{Z}$. The affine representation $\rho: H_{k} \rightarrow \operatorname{Aff}\left(\mathbb{R}^{3}\right)$ can be extended to $\tilde{\rho}: E: \operatorname{Aff}\left(\mathbb{R}^{3}\right)$ by setting

$$
\tilde{\rho}(\alpha)=\left(\begin{array}{cccc}
1 & l & m & \frac{n}{2} \\
0 & -1 & 0 & 0 \\
0 & 0 & -1 & 0 \\
0 & 0 & 0 & 1
\end{array}\right)
$$

(see 9 , p. 160]). The computation of the polynomial $\tilde{\rho}(E)$-invariant forms closely follows the lines drawn in Example 3.3 , resulting in the following cohomology spaces:

$$
\begin{array}{ll}
H_{\text {triv }}^{0}(E, \mathbb{R}) \cong \mathbb{R}, & H_{\text {triv }}^{1}(E, \mathbb{R}) \cong 0, \\
H_{\text {triv }}^{2}(E, \mathbb{R}) \cong 0, & H_{\text {triv }}^{3}(E, \mathbb{R}) \cong \mathbb{R} .
\end{array}
$$

Example 3.5 continued. Let us have a look at the extension $F_{2,4} \rtimes \mathbb{Z}_{4}$ of the free 4-generated 2-step nilpotent group by $\mathbb{Z}_{4}=\left\langle t \mid t^{4}=1\right\rangle$, where

$$
\begin{aligned}
& t e_{1}=e_{2} t, \quad t e_{2}=e_{3} t, \quad t e_{3}=e_{4} t, \quad t e_{4}=e_{1} t, \quad t e_{5}=e_{8} t, \\
& t e_{6}=e_{9} t, \quad t e_{7}=e_{5}^{-1} t, \quad t e_{8}=e_{10} t, \quad t e_{9}=e_{6}^{-1} t, \quad t e_{10}=e_{7}^{-1} t .
\end{aligned}
$$


Note that this group has torsion, but is still orientable. Its cohomology is

$$
\begin{array}{lll}
H_{\text {triv }}^{0}(G, \mathbb{R}) \cong \mathbb{R}, & H_{\text {triv }}^{1}(G, \mathbb{R}) \cong \mathbb{R}, & H_{\text {triv }}^{2}(G, \mathbb{R}) \cong \mathbb{R}^{5}, \\
H_{\text {triv }}^{3}(G, \mathbb{R}) \cong \mathbb{R}^{15}, & H_{\text {triv }}^{4}(G, \mathbb{R}) \cong \mathbb{R}^{21}, & H_{\text {triv }}^{5}(G, \mathbb{R}) \cong \mathbb{R}^{22}, \\
H_{\text {triv }}^{6}(G, \mathbb{R}) \cong \mathbb{R}^{21}, & H_{\text {triv }}^{7}(G, \mathbb{R}) \cong \mathbb{R}^{15}, & H_{\text {triv }}^{8}(G, \mathbb{R}) \cong \mathbb{R}^{5}, \\
H_{\text {triv }}^{9}(G, \mathbb{R}) \cong \mathbb{R}, & H_{\text {triv }}^{10}(G, \mathbb{R}) \cong \mathbb{R} . &
\end{array}
$$

4.2. Cohomological duality. When a compact $K(E, 1)$-manifold $Y$ exists for a group $E$, we can consider $Y$ as a quotient manifold resulting from an action of $E$ on the universal covering space $X$ of $Y$. In this situation $X$ is orientable. The morphism $\varphi: E \rightarrow \operatorname{Aut}(\mathbb{R})$ given by

$$
\varphi(e)= \begin{cases}+1 & \text { if the action of } e \text { on } X \text { is orientation-preserving } \\ -1 & \text { if the action of } e \text { on } X \text { is orientation-reversing }\end{cases}
$$

defines the orientation module structure of $\mathbb{R}$. In case $X=\mathbb{R}^{n}$ for some $n$, a diffeomorphism $\lambda$ is orientation-preserving iff $\operatorname{det}[\operatorname{Jac}(\lambda)(x)]>0$ for all $x \in \mathbb{R}^{n}$, and orientation reversing iff $\operatorname{det}[\operatorname{Jac}(\lambda)(x)]<0$ for all $x \in \mathbb{R}^{n}$ (see [15]). However, in case $E$ is a virtually nilpotent group acting on $\mathbb{R}^{n}$ via $\rho: E \rightarrow P\left(\mathbb{R}^{n}\right)$, the quotient space need not be a manifold. Defining $\varphi: E \rightarrow \operatorname{Aut}(\mathbb{R})$ by

$$
\varphi(e)=\operatorname{det}[\operatorname{Jac}(\rho(e))(0)]
$$

still yields a group action, and gives $\mathbb{R}$ an orientation-like module structure. Results about (co)homological duality analoguous to ours can be found in for instance [5].

Theorem 4.5. Let $E$ be a virtually nilpotent group of Hirsch length $n$ and let $N$ be a normal $\mathcal{J}$-subgroup of finite index. Let $E$ act on $\mathbb{R}^{n}$ via $\rho: E \rightarrow \mathcal{D}\left(\mathbb{R}^{n}\right)$ such that the $N$-action is free and properly discontinuous. Let $\varphi: E \rightarrow \operatorname{Aut}(\mathbb{R})$ denote the orientation module structure of $\mathbb{R}$. Then

$$
H_{\text {triv }}^{p}(E, \mathbb{R}) \cong H_{\varphi}^{n-p}(E, \mathbb{R})
$$

for every $p \in\{0,1, \ldots, n\}$.

The following lemma simplifies the proof of the theorem.

Lemma 4.6. Let $F$ be a finite group acting on a vector space $V$ over a field $K$ that is uniquely divisible by the index $|F|$ of $F$. Then

$$
\operatorname{Hom}_{K}(V, K)^{F} \cong \operatorname{Hom}_{K}\left(V^{F}, K\right)
$$

where the $F$-action on $\operatorname{Hom}_{K}(V, K)$ is given by

$$
{ }^{f} \chi: V \rightarrow K: v \mapsto \chi\left({ }^{f^{-1}} v\right)
$$

for $f \in F$ and $\chi \in \operatorname{Hom}_{K}(V, K)$.

Proof of Lemma 4.6. For a $\chi \in \operatorname{Hom}_{K}(V, K)^{F}$ we define $R(\chi) \in \operatorname{Hom}_{K}\left(V^{F}, K\right)$ as the restriction of $\chi$ to $V^{F}$. This is evidently a linear operator. Suppose $R(\chi)(v)=0$ for all $v \in V^{F}$. Then

$$
\chi\left(\sum_{f \in F}{ }^{f} w\right)=0
$$


for all $w \in V$, implying that

$$
\chi(w)=-\sum_{f \in F, f \neq 1} \chi\left({ }^{f} w\right)=-(|F|-1) \chi(w),
$$

so $\chi$ is in fact the zero linear form, and $R$ is injective.

To show $R$ is also surjective, consider a $\xi \in \operatorname{Hom}_{K}\left(V^{F}, K\right)$ and define

$$
\tilde{\xi}: V \rightarrow K: v \mapsto \frac{1}{|F|} \xi\left(\sum_{f \in F}{ }^{f} v\right) .
$$

Then $\tilde{\xi}$ is a $F$-invariant linear form on $V$ such that

$$
R(\tilde{\xi})(v)=\frac{1}{|F|} \xi\left(\sum_{f \in F}{ }^{f} v\right)=\xi(v)
$$

for every $v \in V^{F}$.

Proof of Theorem 4.5. Let $F=E / N$ be finite. Then we know that

$$
H_{\text {triv }}^{*}(E, \mathbb{R}) \cong H_{d R}^{*}\left(\rho(N) \backslash \mathbb{R}^{n}\right)^{F} .
$$

The Poincaré duality theorem (see [15]) states that

$$
H_{d R}^{p}\left(\rho(N) \backslash \mathbb{R}^{n}\right) \cong \operatorname{Hom}_{\mathbb{R}}\left(H_{d R}^{n-p}\left(\rho(N) \backslash \mathbb{R}^{n}\right), \mathbb{R}\right) .
$$

This isomorphism is the mapping induced on cohomology by the map

$$
D: \Omega^{p}\left(\rho(N) \backslash \mathbb{R}^{n}\right) \rightarrow \operatorname{Hom}_{\mathbb{R}}\left(\Omega^{n-p}\left(\rho(N) \backslash \mathbb{R}^{n}\right), \mathbb{R}\right)
$$

defined by

$$
D(\omega)(\eta)=\int_{M} \omega \wedge \eta
$$

for every $\omega \in \Omega^{p}\left(\rho(N) \backslash \mathbb{R}^{n}\right)$ and $\eta \in \Omega^{n-p}\left(\rho(N) \backslash \mathbb{R}^{n}\right)$. This description of the isomorphism allows us to determine the $F$-action on $\operatorname{Hom}_{\mathbb{R}}\left(H_{d R}^{n-p}\left(\rho(N) \backslash \mathbb{R}^{n}\right), \mathbb{R}\right)$. Let $f \in F$ and $e \in E$ such that $e N=f$. Then

$$
\begin{aligned}
D\left({ }^{f} \omega\right)(\eta) & =\int_{\rho(N) \backslash \mathbb{R}^{n}}\left(\rho\left(e^{-1}\right)^{*} \omega\right) \wedge \eta \\
& =\int_{\rho(N) \backslash \mathbb{R}^{n}} \rho\left(e^{-1}\right)^{*}\left(\omega \wedge \rho(e)^{*} \eta\right) \\
& =\int_{\rho\left(e^{-1}\right) \rho(N) \backslash \mathbb{R}^{n}} \omega \wedge \rho(e)^{*} \eta \\
& =\varphi\left(e^{-1}\right) \int_{M} \omega \wedge \rho(e)^{*} \eta \\
& =\mathcal{D}(\omega)\left(\varphi\left(e^{-1}\right) \rho(e)^{*} \eta\right) .
\end{aligned}
$$

This means the action of $F$ on $\operatorname{Hom}_{\mathbb{R}}\left(H_{d R}^{n-p}\left(\rho(N) \backslash \mathbb{R}^{n}\right), \mathbb{R}\right)$ is the dual action of the $F$-action on $H_{d R}^{n-p}\left(\rho(N) \backslash \mathbb{R}^{n}\right)$ induced by

$$
{ }^{f} \eta=\varphi(e) \rho\left(e^{-1}\right)_{*} \eta
$$

for $e \in E$ such that $e N=f$. The lemma above together with Lemma 4.4 now assure that

$$
H_{\text {triv }}^{p}(E, \mathbb{R}) \cong H_{\varphi}^{n-p}(E, \mathbb{R})
$$




\section{Cohomology of Virtually abelian groups}

Let $E$ be an infinite virtually abelian group. Then there exists an $n \in \mathbb{N}_{0}$ and a finite group $F$ such that $E$ is an extension of $\mathbb{Z}^{n}$ by $F$,

$$
1 \rightarrow \mathbb{Z}^{n} \rightarrow E \rightarrow F \rightarrow 1
$$

Such an extension induces an action $\psi: F \rightarrow G L\left(\mathbb{Z}^{n}\right)$ of $F$ on $\mathbb{Z}^{n}$ by conjugation inside $E$. Now let $m: F \times F \rightarrow \mathbb{Z}^{n}$ be the cocycle corresponding to this extension. Then $E$ is isomorphic to the group $\mathbb{Z}^{n} \times_{m} F$ with underlying set $\mathbb{Z}^{n} \times F$ and operation given by

$$
(z, f) \cdot\left(z^{\prime}, f^{\prime}\right)=\left(z+\psi(f) z^{\prime}+m\left(f, f^{\prime}\right), f f^{\prime}\right) .
$$

Considered as a mapping from $F \times F$ to $\mathbb{R}^{n}, m$ is a cocycle as well. But $H^{2}\left(F, \mathbb{R}^{n}\right)$ is trivial for $F$ is finite and $\mathbb{R}^{n}$ is uniquely divisible by the index of $F$, so $m$ is actually a coboundary. Let $\lambda: F \rightarrow \mathbb{R}^{n}$ be a mapping such that $\delta \lambda=m$, that is,

$$
\delta \lambda\left(f, f^{\prime}\right)=\lambda(f)+\psi(f) \lambda\left(f^{\prime}\right)-\lambda\left(f f^{\prime}\right)=m\left(f, f^{\prime}\right)
$$

for all $f, f^{\prime} \in F$.

Theorem 5.1 (With notations as introduced above). The mapping $\rho: E=\mathbb{Z}^{n} \times_{m}$ $F \rightarrow \operatorname{Aff}(n, \mathbb{R})$ given by

$$
\rho(z, f)=\left(\begin{array}{ccc|c} 
& \psi & & z+\lambda(f) \\
& & & \\
\hline 0 & \cdots & 0 & 1
\end{array}\right)
$$

is a group homomorphism whose restriction to $\mathbb{Z}^{n}$ yields an affine crystallographic action.

Proof. Let $(z, f),\left(z^{\prime}, f^{\prime}\right) \in E$. Then

$$
\begin{aligned}
\rho\left((z, f) \cdot\left(z^{\prime}, f^{\prime}\right)\right) & =\rho\left(\left(z+\psi(f) z^{\prime}+m\left(f, f^{\prime}\right), f f^{\prime}\right)\right) \\
& =\left(\begin{array}{c|c}
\psi\left(f f^{\prime}\right) & z+\psi(f) z^{\prime}+m\left(f f^{\prime}\right)+\lambda\left(f f^{\prime}\right) \\
\hline 0 & 1
\end{array}\right)
\end{aligned}
$$

while

$$
\begin{aligned}
& \left(\begin{array}{c|c}
\psi(f) & z+\lambda(f) \\
\hline 0 & 1
\end{array}\right)\left(\begin{array}{c|c}
\psi\left(f^{\prime}\right) & z^{\prime}+\lambda\left(f^{\prime}\right) \\
\hline 0 & 1
\end{array}\right) \\
& =\left(\begin{array}{c|c}
\psi(f) \psi\left(f^{\prime}\right) & z+\psi(f) z^{\prime}+\lambda(f)+\psi(f) \lambda\left(f^{\prime}\right) \\
\hline 0 & 1
\end{array}\right) .
\end{aligned}
$$

Now (5.1) equals (5.2) because $\psi$ is a group homomorhism and

$$
\lambda(f)+\psi(f) \lambda\left(f^{\prime}\right)=\delta \lambda\left(f, f^{\prime}\right)+\lambda\left(f f^{\prime}\right)=m\left(f, f^{\prime}\right)+\lambda\left(f f^{\prime}\right),
$$

so $\rho$ is a group homomorphism. The action of $z \in \mathbb{Z}^{n} \subset E$ under $\rho$ is just translation with $z$, so the restriction of $\rho$ to $\mathbb{Z}^{n}$ is injective and the action of $\mathbb{Z}^{n}$ on $\mathbb{R}^{n}$ is free and properly discontinuous.

From Example 3.2 we already know that $H^{*}\left(\mathbb{Z}^{n}, \mathbb{R}\right) \cong \operatorname{Alt}^{*}\left(\mathbb{R}^{n}\right)$, the graded $\mathbb{R}$ algebra of alternating forms on $\mathbb{R}^{n}$. Now let $\operatorname{Alt}^{*}\left(\mathbb{R}^{n}\right)^{\psi(F)}$ be the graded $\mathbb{R}$-algebra of $\psi(F)$-invariant alternating forms on $\mathbb{R}^{n}$, that is, the space of all $\omega \in A l t^{p}\left(\mathbb{R}^{n}\right)$ 
satisfying

$$
\omega\left(\xi^{(1)}, \ldots, \xi^{(p)}\right)=\omega\left(\psi(f) \xi^{(1)}, \psi(f) \xi^{(2)}, \ldots, \psi(f) \xi^{(p)}\right)
$$

for any $f \in F$ and $\xi^{(1)}, \ldots, \xi^{(p)} \in \mathbb{R}^{n}$. Then Theorem 4.3 can be stated as follows.

Theorem 5.2. $H^{*}(E, \mathbb{R}) \cong \operatorname{Alt}^{*}\left(\mathbb{R}^{n}\right)^{\psi(F)}$.

There is an immediate way to compute the rank of $H^{*}(E, \mathbb{R})$ for every $p$. The formula uses the following notation. Let $n, p \in \mathbb{N}_{0}$ and let $A=\left(a_{i j}\right) \in \mathbb{R}^{n \times n}$ be a matrix. For every couple of $(p, n-p)$-shuffles $(\sigma, \tau)$ we set

$$
A_{(\sigma, \tau)}=\operatorname{det}\left(\begin{array}{cccc}
a_{\sigma(1) \tau(1)} & a_{\sigma(1) \tau(2)} & \ldots & a_{\sigma(1) \tau(p)} \\
a_{\sigma(2) \tau(1)} & a_{\sigma(2) \tau(2)} & \ldots & a_{\sigma(2) \tau(p)} \\
\vdots & \vdots & \ddots & \vdots \\
a_{\sigma(p) \tau(1)} & a_{\sigma(p) \tau(2)} & \ldots & a_{\sigma(p) \tau(p)}
\end{array}\right) .
$$

Listing all $(p, n-p)$-shuffles as

$$
\sigma_{1}, \sigma_{2}, \ldots, \sigma_{c} \quad \text { with } c=\left(\begin{array}{c}
n \\
p
\end{array}\right)
$$

we can define

$$
A^{(p)}=\left(\begin{array}{cccc}
A_{\left(\sigma_{1}, \sigma_{1}\right)} & A_{\left(\sigma_{1}, \sigma_{2}\right)} & \ldots & A_{\left(\sigma_{1}, \sigma_{c}\right)} \\
A_{\left(\sigma_{2}, \sigma_{1}\right)} & A_{\left(\sigma_{2}, \sigma_{2}\right)} & \ldots & A_{\left(\sigma_{2}, \sigma_{c}\right)} \\
\vdots & \vdots & \ddots & \vdots \\
A_{\left(\sigma_{c}, \sigma_{1}\right)} & A_{\left(\sigma_{c}, \sigma_{2}\right)} & \ldots & A_{\left(\sigma_{c}, \sigma_{c}\right)}
\end{array}\right)
$$

The following proposition gives explicit formulas for the computation of all Betti numbers $\beta_{p}(E)$ of a virtually abelian group $E$.

Proposition 5.3 (With notations as above). Let $F$ be generated by $a_{1}, a_{2}, \ldots, a_{r}$. Then

$$
\beta_{p}(E)=\left(\begin{array}{c}
n \\
p
\end{array}\right)-\operatorname{Rank}\left(\psi\left(a_{1}\right)^{(p)}-\mathbb{I}, \ldots, \psi\left(a_{r}\right)^{(p)}-\mathbb{I}\right) .
$$

Moreover, let $\varphi: F \rightarrow \operatorname{Aut}(\mathbb{R})$ be the morphism indicating the orientation module structure of $\mathbb{R}$ determined by $\rho$. Then

$$
\beta_{p}(E)=\left(\begin{array}{c}
n \\
p
\end{array}\right)-\operatorname{Rank}\left(\varphi\left(a_{1}\right) \psi\left(a_{1}\right)^{(n-p)}-\mathbb{I}, \ldots, \varphi\left(a_{r}\right) \psi\left(a_{r}\right)^{(n-p)}-\mathbb{I}\right) .
$$

The matrix of which we take the rank in this proposition is obtained by juxtaposition of the matrices in the formula, the matrix $\mathbb{I}$ is the identity matrix of appropriate dimension. The proof of Proposition 5.3 uses the lemma below. The proof of the lemma is left to the reader.

Lemma 5.4. Let $\sigma \in \mathcal{S}(p, n-p)$ and $f \in F$. Then

${ }^{f}\left(d x_{\sigma(1)} \wedge d x_{\sigma(2)} \wedge \ldots \wedge d x_{\sigma(p)}\right)=\sum_{\tau \in \mathcal{S}(p, n-p)} \psi\left(f^{-1}\right)_{(\sigma, \tau)} d x_{\tau(1)} \wedge d x_{\tau(2)} \wedge \ldots \wedge d x_{\tau(p)}$.

Proof of Proposition 5.3. We will prove both formulas simultanuously, using the $F$-action on differential forms given by

$$
{ }^{f} \omega=\varphi(e) \rho\left(e^{-1}\right)^{*} \omega
$$


for all $\omega \in \Omega^{*}\left(\mathbb{R}^{n}\right), f \in F$ and $e \in E$ such that $e N=f$. For the first formula choose $\varphi: E \rightarrow \operatorname{Aut}(\mathbb{R})$ to be trivial and use Theorem [5.2, and for the second let $\varphi$ be the orientation module structure and use Theorem 4.5.

Let $\omega \in \operatorname{Alt}^{p}\left(\mathbb{R}^{n}\right)$. Considering $\omega$ as a constant $p$-form on $\mathbb{R}^{n}$ there is an $\omega_{\sigma} \in \mathbb{R}$ for every $\sigma \in \mathcal{S}(p, n-p)$ such that

$$
\omega=\sum_{\sigma \in \mathcal{S}(p, n-p)} \omega_{\sigma} d x_{\sigma(1)} \wedge d x_{\sigma(2)} \wedge \ldots \wedge d x_{\sigma(p)} .
$$

We are interested in the $F$-invariant differential forms. Computing the action of $f \in F$ on $\omega$ using the lemma above yields

$$
\begin{aligned}
{ }^{f} \omega & =\varphi(f) \sum_{\sigma \in \mathcal{S}(p, n-p)} \omega_{\sigma} \psi\left(f^{-1}\right)^{*}\left(d x_{\sigma(1)} \wedge d x_{\sigma(2)} \wedge \ldots \wedge d x_{\sigma(p)}\right) \\
& =\varphi(f) \sum_{\sigma \in \mathcal{S}(p, n-p)} \omega_{\sigma} \sum_{\tau \in \mathcal{S}(p, n-p)} \psi\left(f^{-1}\right)_{(\sigma, \tau)} d x_{\tau(1)} \wedge d x_{\tau(2)} \wedge \ldots \wedge d x_{\tau(p)} .
\end{aligned}
$$

This differential form must equal $\omega$, which means that

$$
\varphi(f) \sum_{\sigma \in \mathcal{S}(p, n-p)} \psi\left(f^{-1}\right)_{(\sigma, \tau)} \omega_{\sigma}=\omega_{\tau}
$$

for all $\tau \in \mathcal{S}(p, n-p)$. So the differential forms that are invariant under the action of $f$ are the solutions of a linear set of homogeneous equations with matrix $\varphi(f) \psi\left(f^{-1}\right)^{(p)}-\mathbb{I}$.

Of course, it is enough that $\omega$ be invariant under a set of generators $\left\{a_{1}, a_{2}, \ldots\right.$, $a_{r}$ \} of $F$. Also note that $\varphi(f)=\varphi\left(f^{-1}\right)$, so the $F$-invariant differential forms are simultanuously a solution to the sets of homogeneous linear equations given by the matrices

$$
\varphi\left(a_{1}\right) \psi\left(a_{1}\right)^{(p)}-\mathbb{I}, \ldots, \varphi\left(a_{r}\right) \psi\left(a_{r}\right)^{(p)}-\mathbb{I} .
$$

As an example, we have computed the Betti numbers for all 5-dimensional Bieberbach groups using Proposition 5.3. The table below lists all possible Betti number configurations. We are very much in debt to the CARAT-team for having provided the representations via their website http://momo.math.rwthaachen.de/carat/.

\begin{tabular}{|c|c|c|c|c|c|}
\hline$\beta_{0}$ & $\beta_{1}$ & $\beta_{2}$ & $\beta_{3}$ & $\beta_{4}$ & $\beta_{5}$ \\
\hline 1 & 0 & 0 & 0 & 0 & 1 \\
1 & 0 & 0 & 1 & 0 & 0 \\
1 & 0 & 0 & 2 & 1 & 0 \\
1 & 0 & 1 & 1 & 0 & 1 \\
1 & 0 & 1 & 2 & 0 & 0 \\
1 & 0 & 1 & 3 & 1 & 0 \\
1 & 0 & 2 & 4 & 1 & 0 \\
1 & 0 & 3 & 3 & 0 & 1 \\
1 & 1 & 0 & 0 & 0 & 0 \\
1 & 1 & 0 & 0 & 1 & 1 \\
1 & 1 & 0 & 1 & 1 & 0 \\
1 & 1 & 1 & 1 & 0 & 0 \\
& & & & &
\end{tabular}

\begin{tabular}{|l|c|c|c|c|c|}
1 & 1 & 1 & 3 & 2 & 0 \\
1 & 1 & 2 & 2 & 1 & 1 \\
1 & 1 & 3 & 3 & 0 & 0 \\
1 & 1 & 4 & 4 & 1 & 1 \\
1 & 1 & 6 & 6 & 1 & 1 \\
1 & 2 & 1 & 0 & 0 & 0 \\
1 & 2 & 1 & 1 & 2 & 1 \\
1 & 2 & 2 & 2 & 1 & 0 \\
1 & 2 & 4 & 6 & 3 & 0 \\
1 & 3 & 3 & 1 & 0 & 0 \\
1 & 3 & 4 & 4 & 3 & 1 \\
1 & 4 & 6 & 4 & 1 & 0 \\
1 & 5 & 10 & 10 & 5 & 1 \\
\hline
\end{tabular}


Remark. It is clear from the above formulas that once the action $\psi$ of $F$ on $\mathbb{Z}^{n}$ is fixed, the cocycle that determines the extension $E$ does not influence the rank of the cohomology groups. Moreover, looking at the invariance conditions we can see that the cocycle will not influence the actual cohomology space. Of course this is no longer true for integral cohomology groups.

Remark. One can easily see from the invariance condition that every action of $F$ on $\mathbb{Z}^{n}$ that is conjugated inside $G L(n, \mathbb{R})$ to a given action will give rise to the same Betti numbers. In this case the actual structure of the cohomology spaces will be isomorphic.

\section{REFERENCES}

[1] Auslander, L. and Markus, L. Holonomy of Flat Affinely Connected Manifolds. Ann. of Math., 1955, 62 (1), pp. 139-151. MR0072518 (17:298b)

[2] Babakhanian, A. Cohomological methods in group theory, volume 11 of Pure and Applied Mathematics. Marcel Dekker Inc., New York, 1972.

[3] Benoist, Y. Une nilvariété non affine. J. Differential Geom., 1995, 41 pp. 21-52. MR.1316552 (96c:53077)

[4] Benoist, Y. and Dekimpe, K. The Uniqueness of Polynomial Crystallographic Actions. Math. Ann., 2002, 322 (2), pp. 563-571. MR1895707 (2003a:20078)

[5] Bieri, R. Gruppen mit Poincaré Dualität. Comment. Math. Helv., 1972, 47 pp. 373 - 396. MR0352290 (50:4777)

[6] Brown, K. S. Cohomology of groups, volume 87 of Grad. Texts in Math. Springer-Verlag, New York, Inc., 1982. MR0672956 (83k:20002)

[7] Burde, D. Affine structures on nilmanifolds. Internat. J. Math, 1996, 7 (5) pp. 599 - 616. MR1411303 (97i:53056)

[8] Burde, D. and Grunewald, F. Modules for certain Lie algebras of maximal class. J. Pure Appl. Algebra, 1995, 99 pp. 239-254. MR:1332900 (96d:17007)

[9] Dekimpe, K. Almost-Bieberbach Groups: Affine and Polynomial Structures, volume 1639 of Lect. Notes in Math. Springer-Verlag, 1996. MR1482520 (2000b:20066)

[10] Dekimpe, K. and Igodt, P. Polycyclic-by-finite groups admit a bounded-degree polynomial structure. Invent. Math., 1997, 129 (1) pp. 121-140. MR.1464868 (99c:20071)

[11] Dekimpe, K. and Igodt, P. Polynomial structures on polycyclic groups. Trans. Amer. Math. Soc., 1997, 349 pp. 3597-3610. MR1422895 (98f:20021)

[12] Dekimpe, K., Igodt, P., and Lee, K. B. Polynomial structures for nilpotent groups. Trans. Amer. Math. Soc., 1996, 348 pp. 77-97. MR1327254 (96e:20051)

[13] Fried, D., Goldman, W., and Hirsch, M. Affine manifolds with nilpotent holonomy. Comment. Math. Helv., 1981, 56 pp. 487-523. MR0656210(83h:53062)

[14] Hochschild, G. and Serre, J.-P. Cohomology of Group Extensions. Trans. Amer. Math. Soc., 1953, 74 pp. 110-134. MR0052438 (14:619b)

[15] Madsen, I. and Tornehave, J. From Calculus to Cohomology. Cambridge University Press, 1997. MR 1454127 (98g:57040)

[16] Massey, W. S. Singular Homology Theory, volume 70 of Grad. Texts in Math. SpringerVerlag, 1980. MR.0569059 (81g:55002)

[17] Nomizu, K. On the cohomology of compact homogeneous spaces of nilpotent Lie groups. Ann. of Math., 1954, 59 pp. 531-538. MR0064057 (16:219c)

[18] Raghunathan, M. S. Discrete Subgroups of Lie Groups, volume 68 of Ergebnisse der Mathematik und ihrer Grenzgebiete. Springer-Verlag, 1972. MR0507234 (58:22394a)

[19] Segal, D. Polycyclic Groups. Cambridge University Press, 1983. MR0713786 (85h:20003)

[20] Tangora, M. C. ed. Computers in geometry and topology, volume 114 of Lecture notes in pure and applied mathematics. Marcel Dekker, Inc., 1989. MR0988688 (89j:55002)

Katholieke Universiteit Leuven, Campus Kortrijk, B-8500 Kortrijk, Belgium

Katholieke Universiteit Leuven, Campus Kortrijk, B-8500 Kortrijk, Belgium Current address: Gelÿkmeidstraat 12/2, B-8400 Oostende, Belgium 